\title{
Modern Stereochemistry
}

$\mathrm{O}^{\mathrm{N}}$

$\mathrm{NE}$ of the fundamental contributions of chemistry to our knowledge of the properties of atoms is the conception of discrete valency bonds with definite directions in space. This conception rests upon an immense mass of experimental evidence, and its principal features must be retained in the new pictures of atoms and molecules which are now being developed on the basis of modern physical theories. The relation between the old and the new atom models was a notable feature of the discussion on "Stereochemistry" held by the Chemical Society on January 16.

Dr. W. H. Mills opened the discussion with a brief survey of the success of the old tetrahedral model of the carbon atom. It accounts in simple fashion for the dissymmetry of compounds of the type Cabcd, for the lack of free rotation about a double bond, and for the linear configuration of acetylene and other compounds containing a triple bond. A further deduction from the tetrahedral model is the dissymmetry of allene derivatives of the type

$$
\begin{aligned}
& a \backslash \mathrm{C}: \mathrm{C}: \mathrm{C}^{\prime} / c \\
& d
\end{aligned}
$$

The possibility of optical activity in these compounds was predicted by van't Hoff in 1875 ; the isolation of allene derivatives in optically active forms was first achieved by Mills and Maitland in 1935.

Dr. Maitland later in the discussion gave experimental details of this very interesting work. From the tetrahedral model it is readily seen that four different groups attached to the allene residue are not necessary. Compounds of the type $\begin{aligned} & a \\ & b\end{aligned}>\mathrm{C}: \mathrm{C}: \mathrm{C}<\begin{aligned} & a \\ & b\end{aligned}$ should also be resolvable since the plane passing through $\mathrm{Cab}$ at one end of the molecule is at right angles to the plane through $\mathrm{Cab}$ at the other end. After a great deal of fruitless work, success was

$\mathrm{OH}$

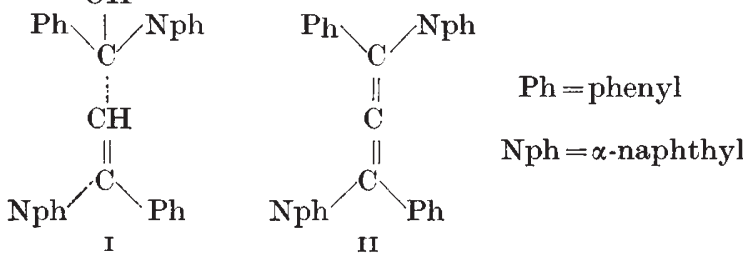

attained with the allene derivative II, which was prepared by dehydrating the alcohol, I, with $d$-or $l$-camphorsulphonic acid in dilute benzene solution. Most of the product was the racemic form, but the use of an asymmetric dehydrating agent gave about five per cent of the optically active hydrocarbon. Its high rotation $\left([\alpha]_{5461} \pm 438^{\circ}\right)$ revealed its presence in the mixture, and its solubility relations allowed it to be separated from the inactive form and to be isolated in an optically pure condition; $d$-camphorsulphonic acid produced the $d$ form of the allene whilst the $l$-acid gave the $l$-allene.

Dr. Mills then briefly described thepictures of valency bonds which are based on quantum mechanics, and in particular referred to the model of a double bond. In this model the shared electrons moving in $s$ levels (that is, levels with serial quantum number $l=0$ ) form an electron cloud symmetrical about the line joining the carbon atoms, whilst the electrons in $p$ levels (with $l=1$ ) may be regarded in the first place as moving in paths for which wave-mechanics gives a roughly dumb-bell-shaped electron cloud normal to the carbon-carbon axis. Interaction between the $p$ electrons on adjacent carbon atoms constrains these dumb-bell-shaped figures to remain parallel to one another as shown in III, and thus accounts for the lack of free rotation about a double bond. In the allene derivatives, as Iv shows, the central carbon atom is associated with two sets of $p$ electron clouds at right angles to one another; these by interaction with the $p$ clouds on the terminal carbon atoms lock the valency bonds of these atoms into definite space directions.
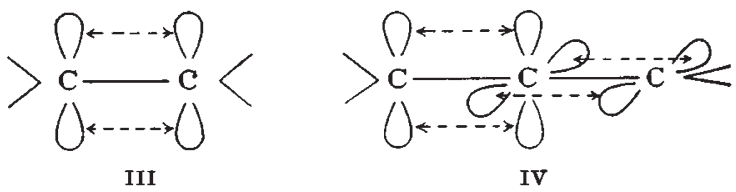

Another application of wave-mechanics to a stereo. chemical problem concerns derivatives of triphenylmethyl. On the theory developed by Hückel (1933), the stability of a free radical of the type $\mathrm{CAr}_{3}$ depends upon wave-mechanical resonance between three aryl radicals arranged at angles of $120^{\circ}$ in the plane around the central carbon atom. Such an arrangement is obviously incompatible with the existence of an optically active free radical, even if three different aryl groups are attached to the central carbon atom. In accordance with this model, Wallis and Adam (1933) have found that whilst the ions $\mathrm{CAr}_{3}$ - and $\mathrm{CAr}_{3}+$ (which contain one electron more or less than the free radical) can exist in optically active forms, the activity is lost when they pass into the free radicals.

Another type of reaction in which an optically active $\mathrm{CR}_{3}+$ ion may exist for a short time was discussed by Prof. A. McKenzie. The optically active substance $\mathrm{v}$, when treated with nitrous acid, passes smoothly into the ketone vIrI, which is also optically active. The probable course of the reaction is shown by the formulæ $\mathrm{V}$ to VIII, in which the asymmetric carbon is indicated by an asterisk. When nitrogen and water split off from vI, the intermediate product vI<smiles>CC(C(=O)NO)C(O)(c1ccccc1)c1ccccc1</smiles>
$\mathrm{v}$

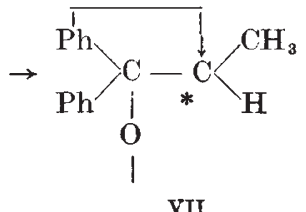
VII<smiles>C[C@H]([N+]=O)C(O)(c1ccccc1)c1ccccc1</smiles>

VI<smiles>CC(C(=O)c1ccccc1)C(C)c1ccccc1</smiles>

$[\alpha]_{5461}+211^{\circ}$
VIII 
has a positive charge on the carbon atom. This unstable entity passes into viII by the migration of a phenyl group as shown by the arrow in formula vir. Since the product is strongly active, it is clear that the intermediate carbonium ion in VII cannot possess a planar configuration. Similar migrations of radicals occur when glycols are dehydrated. With some optically active glycols, the ketone produced is also optically active, as in the production of $\mathrm{x}$ from IX ; in other cases the product is completely inactive.

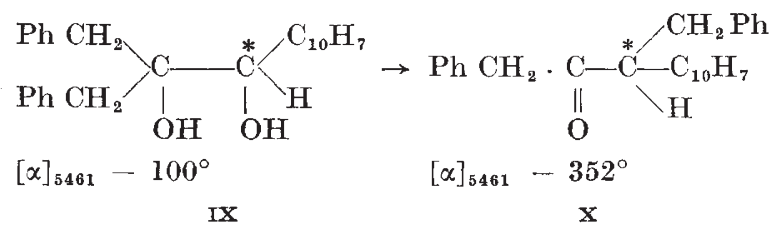

The valency links which have so far been discussed are described by the quantum theory as compounded of electrons in $s$ and $p$ levels. An interesting feature of the theory of Pauling (1931) is the configuration deduced for atoms in which $d$ levels (with $l=2$ ) are compounded with $s$ and $p$ levels to form valency bonds. When one $d$ level is used, the theory predicts an arrangement of four bonds at $90^{\circ}$ in one plane. Vacant $d$ levels with energies comparable with $s$ and $p$ levels are found in the atoms of transition elements ; with certain of these the utilisation of a $d$ level to form the valency bond should alter the magnetic moment of the metallic atom. Thus, for example, bivalent nickel, which is paramagnetic in its simple salts, should become diamagnetic in co-ordination compounds in which it has a planar configuration.

Recent work on compounds of this type was reviewed by Prof. S. Sugden. There are now three main lines of evidence in favour of a planar configuration of compounds of nickel, palladium and platinum. First there is the occurrence of cis-trans isomerides when two unsymmetrical chelate groups are attached to the metallic atom. As an example, the isomeric nickel derivatives of benzylmethylglyoxime (Sugden, 1932) shown in formulæ XI and xII may be quoted. Other examples are furnished

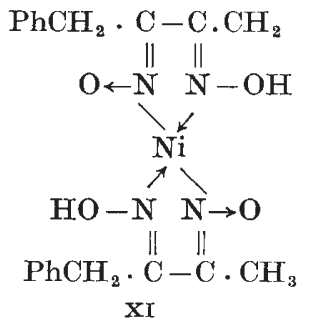

by the isomeric glycine derivatives of platinum (Grünberg and Ptizyn, 1933) and of palladium (Pinkard, Sharrard, Wardlaw, and Cox, 1934).

Evidence from optical activity is provided by the resolution of the compound xur, which was achieved by Mills and Quibell in 1935. If the links to the platinum atom had a tetrahedral arrangement instead of the planar configuration shown in the formula (xur), then the planes of the two rings would be at right angles; the molecule would thus possess a plane of symmetry and the substance should not be resolvable.

Finally, X-ray studies by Cox, Wardlaw and others in the last two years have demonstrated a planar

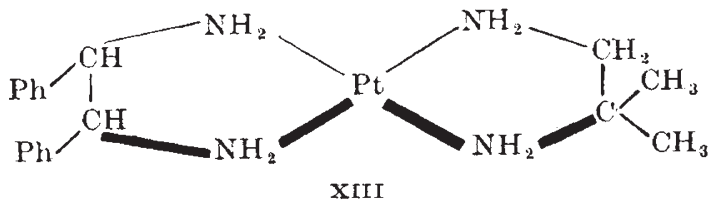

configuration in no less than 14 derivatives of nickel, palladium and platinum. The dithio-oxalate, $x I v$, is of special interest since the good scattering power of the sulphur atoms enabled the position of all the atoms in the molecule to be determined by X-ray analysis.

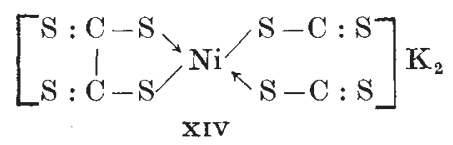

There is thus abundant evidence for the frequent occurrence of a planar configuration in four covalent complexes of these three elements. The magnetic evidence is also in accord with Pauling's theory for nickel, which is diamagnetic in all the compounds which have been shown to be planar but paramagnetic in other simple and complex salts. Since all the compounds of palladium and platinum, whether simple or complex, are found to be diamagnetic, no change in magnetism can be observed, but the diamagnetism of the planar derivatives is in accord with Pauling's views. A real discrepancy appears, however, with cupric compounds. Cox and Webster (1935) have found from $X$-ray studies that the cupric derivatives of certain $\beta$-diketones have a planar configuration. These compounds are paramagnetic, and there is not a vacant $3 d$ level in the cupric ion. Similar difficulties arise in the interpretation of the magnetic data for some octahedral complexes in which Pauling postulates the use of two $d$ levels.

Finally, Dr. E. E. Turner gave a comprehensive survey of the stereochemistry which arises from restricted rotation about a single bond. In diphenyl derivatives of the type $\mathrm{xv}$,

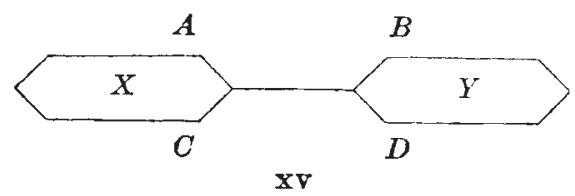

the substituents $A, B, C, D$, if they are large enough, may block the free rotation of the benzene rings $X$ and $Y$ so that they cannot pass through a coplanar configuration. The molecule may then have no plane or centre of symmetry and should be resolvable. This explanation of the optical activity of diphenyl derivatives was given by Mills in 1926; since then a large number of investigations has been concerned with the number and size of the substituent groups necessary to give optical activity, the relation between rate of racemisation and the size of the obstacles, etc. If the substituent groups are large enough, only two or even one substituent is necessary, since it cannot clear the corner of the benzene ring when the two rings are rotated. Another interesting point which was discussed is the part played by the rotation of groups of the type $-\mathrm{N}\left(\mathrm{CH}_{3}\right)_{2}$ about the bond joining them to the benzene ring; this rotation enhances the power of such a group to form an obstacle to the free rotation of the two benzene rings.
S. S. 